\title{
Deep Hierarchical Attention Active Learning for Mental Disorder Unlabeled Data in AloMT
}

\author{
USMAN AHMED and JERRY CHUN-WEI LIN, Western Norway University of Applied Sciences, \\ Norway \\ GAUTAM SRIVASTAVA, Brandon University, Canada and China Medical University, Taiwan
}

In the Artificial Intelligence of Medical Things (AIoMT), Internet-Delivered Psychological Treatment (IDPT) effectively improves the quality of mental health treatments. With the advent of COVID-19, psychological tasks have become overloaded and complicated for medical professionals due to the overlap of sentimental values. The development of an AIoMT tool requires labeling of data to achieve clinical-level performance. Text data requires an appropriate set of linguistic features for vector latent representation and segmentation. Emotional biases could lead to incorrect segmentation of patient-authorized texts, and labeling emotional data is time-consuming. In this article, we propose an assistant tool for psychologists to assist them in mental health treatment and note-taking. We first extend the word and emotion lexicon and then apply a hierarchical attention method to support data labeling. The learned latent representation uses word position prediction and sentence-level attention to create a semantic framework. The augmented vector representation helps in highlighting words and classifying nine different symptoms from the text written by the patient. Our experimental results show that the emotion lexicon helps to increase the accuracy by $5 \%$ without affecting the overall results, and that the hierarchical attention method achieves an F1 score of 0.89 .

CCS Concepts: • Applied computing $\rightarrow$ Health care information systems; $\bullet$ Computing methodologies $\rightarrow$ Neural networks;

Additional Key Words and Phrases: Artificial intelligence of medical things, Internet-Delivered Psychological Treatment, active learning, classification

ACM Reference format:

Usman Ahmed, Jerry Chun-Wei Lin, and Gautam Srivastava. 2023. Deep Hierarchical Attention Active Learning for Mental Disorder Unlabeled Data in AIoMT. ACM Trans. Sensor Netw. 19, 3, Article 49 (February 2023), 18 pages.

https://doi.org/10.1145/3519304

\section{INTRODUCTION}

With the growth of the Internet of Things (IoT), Artificial Intelligence of Things (IoT) in sensor systems has increased health data for monitoring tasks. Sensor networks are needed in IoTbased health systems for development, testing, and trials. With the increase in online methods,

Authors' addresses: U. Ahmed and J. C.-W. Lin (corresponding author), Department of Computer Science, Electrical Engineering and Mathematical Sciences, Western Norway University of Applied Sciences, Inndalsveien 28, 5063 Bergen, Norway; emails: usman.ahmed@hvl.no, jerrylin@ieee.org; G. Srivastava, Department of Math and Computer Science, Brandon University, Brandon, Manitoba, CANADA, R7A 6A9 and Research Centre for Interneural Computing, China Medical University, Taichung 40402, Taiwan; email: SRIVASTAVAG@brandonu.ca.

Permission to make digital or hard copies of all or part of this work for personal or classroom use is granted without fee provided that copies are not made or distributed for profit or commercial advantage and that copies bear this notice and the full citation on the first page. Copyrights for components of this work owned by others than ACM must be honored. Abstracting with credit is permitted. To copy otherwise, or republish, to post on servers or to redistribute to lists, requires prior specific permission and/or a fee. Request permissions from permissions@acm.org.

(C) 2023 Association for Computing Machinery.

1550-4859/2023/02-ART49 \$15.00

https://doi.org/10.1145/3519304 
mental health treatment requires intelligent monitoring of patients. The increase in sensor-based data can help develop effective tools for artificial intelligence of medical things (AIoMT) Predicting the future condition of patients can help medical professionals take preventive measures in diagnosis. This contributes to reliable, time-effective decision making in real time.

According to the recent report of the World Health Organization (WHO), the COVID-19 pandemic has affected mental health care in $93 \%$ of nations. The lockdown of affected regions as a preventive measure has increased the demand for mental health services. The lockdown has increased physiological stressors such as fear of illness and worry about the future [35]. Emotional stress is also caused by social isolation, lack of school interactions, and purpose of employment, all of which contribute to deteriorating public mental health. Due to fear of illness, lack of protective equipment, social isolation, and a stressful workplace, frontline health professionals also suffer from anxiety and depression symptoms [2], and depression is much greater than any term can convey. In essence, it is an unexpected reaction to life. Numerous factors are often associated with its study because there are so many unknowns about what causes anxiety. The extensive and growing literature points in different directions. Depression is not caused by a single immediate problem or event, but by a mixture of current events and longer-term or personal causes [25]. It is not always possible to determine the cause or change in adverse circumstances [17], even though each person is unique. The most important thing is that people notice the signs and symptoms and even seek help [18]. Currently, there are a variety of websites and other media platforms where people can communicate with each other and talk confidentially about their difficulties People around the world can share their thoughts and experiences [24]. Online monitoring has the potential to be a proactive and promising method for identifying high-risk individuals. It has the potential to effect timely referral and improve overall well-being [28].

Depression is one of the most debilitating diseases in the world, according to the WHO [11]. It affects more than 264 million people worldwide. Untreated depression can worsen and cause significant suffering to those affected [20]. Suicide can be the result of anxiety in the worst cases. According to the WHO, nearly 800,000 people die each year as a result of suicide. Among 15-29-year-olds, suicide is the second-leading cause of death. Between $76 \%$ and $85 \%$ of people with serious mental illnesses go undiagnosed in low- and middle-income countries. Lack of funding, a shortage of qualified health workers, misjudgments, and the social stigma associated with mental illness are all barriers to successful treatment [11]. The main barriers that prevent people from seeking treatment are social stigma, hesitation, and fear of disclosing the problem. People are often ashamed, feel humiliated, and are afraid to have their mental health condition examined in depth [26]. Because of these factors, people may be hesitant to admit that they are sad or to seek therapy. The prevention and treatment of mental disorders is a global concern for health systems. Due to economic and technical factors, the overburdened health care system is under pressure to build an adaptable system that reduces wait times and provides interventions at a lower cost. They also delivered Internet-Delivered Psychological Treatment (IDPT), which can help a huge population overcome emotional and physical discomfort while using fewer resources [25]. Most of the current solutions are tunnel based, rigid, and not interoperable. The developed models lack adaptive behavior, resulting in lower user compliance and more dropouts. User acceptance must be considered in such interventions. Such user acceptance can be done by implementing the IDPT system to account for user behavior. This user behavior includes various preferences, environmental requirements, and psychological symptoms.

Another proposed method is DeepHeart, a heart rate estimation method based on spectrum analysis-based calibration [3]. The method uses the convolutional neural network and applies the denoising-based photoplethysmography signals. Then ensemble-based denoising convolutional neural networks (DCNNs) are used to calibrate and estimate the final heart rate. The method 
used the IEEE Signal Processing Cup training data, which contains an approximation of physical activities. The proposed model achieved an average absolute error of 1.61 beats per minute (bpm) compared to other methods.

Another method used the mobile crowdsensing (MCS) method to analyze the playing patterns and behavioral analyses of mobile users [39]. In addition to user behavior analysis, it is proposed to focus on the development of task requester characteristic analysis. This results in an online incentive mechanism based on group purchases to evaluate computational efficiency, truthfulness, and good performance.

The aim of this research is to extract depressive symptoms from texts written by patients. Using the Deep Attention-Based method, we attempt to detect and visualize them. We explore the extraction of elements that cause depression-related symptoms based on patient-produced content. Using an online interactive technology (ICT) interactive technology to provide contextual information and visualizations for good mental health, preventive measures can be taken. In particular, this study makes the following contributions:

(1) We propose a lexicon-based method for semantic vector expansion and clustering to increase the number of instances that can be trained.

(2) We propose attention-based active learning with deep entropy to extract depression symptoms in mental health interventions.

(3) In in-depth experiments, we evaluate the generalization of the learning system by reducing the data annotation duties.

The remainder of the article is organized as follows. Section 2 outlines related work. Section 3 shows the methodology used to set up the experiment, collect data, and build the model. The results and findings are discussed in Section 4. Finally, Section 5 concludes with a summary and recommendations for further research.

\section{RELATED WORK}

Several attempts have been made to improve the diagnosis of depression by computer-aided detection. This section reviews the many techniques presented. Li et al. [15] developed a Computer Adaptive Test (CAT) based on Item Response Theory (IRT) to assess depression symptoms (Depression-CAT, D-CAT). They intended to develop an application that would assess depression symptom intensity using real patient data to improve measurement accuracy and reduce respondent burden. Instead of using a static questionnaire, an adaptive questionnaire was used to achieve progress in measurement. Data from previously answered questions were used to select the next appropriate questions. Focusing on the most important questions for each patient CAT resulted in fewer items and better coverage. Unfortunately, some questions remained unanswered. For example, the effects of changes in the order of questions were unknown. Indeed, it was uncertain whether the different response alternatives within a single test had any effect on response behavior.

$\mathrm{Xu}$ et al. focus on some basic supervised classification algorithms and text-based features for automatic classification of mental affect states in short texts using a small dataset [38]. This method illustrates a binary classification problem in which short sentences are categorized as disturbed or not disturbed. At a finer level, there were four text classes: high distress, mild distress, response, and joy. The annotated dataset of short written texts for the study categorized any post that indicated an active desire to hurt someone or oneself as high distress, while posts that merely described negative sentiments were typically categorized as low distress. The researchers used a dataset of 200 posts from various public Internet forums dealing with mental health. The dataset was subjected to machine learning methods such as Naive Bayes, Maximum Entropy, and Decision 
Tree. When classifying four methods based on the level of distress, they achieved an accuracy of $54.5 \%$ from a baseline of $30.5 \%$.

Dinakar et al. [8] used a stacked generalization modeling technique to study children in online communities under stress. First, they trained a set of given methods for estimating individual labels, including a Support Vector Machine with Linear Kernel (SVM-L), a Radial Basis Function Kernel (SVM-R), and a Stochastic Gradient Boosted Decision Trees (GBDT) model. Unigrams, bigrams, part-of-speech bigrams, and TF-IDF, filtered by chi-squared feature selection and additional hand-coding features, were incorporated into the basic classifiers. The output of the basic classifiers was a vector of predictions. This topic distribution of the L-LDA model for a given story and the decision function scores for each prediction became meta-features for the metalearners. The researchers examined 7,147 personal experiences submitted by teens with problems on a popular teen-help website.

De Choudhury et al. [7] analyzed the behavior of teenagers and Twitter users in general to look for signs of mental illness. The researchers wanted to build a machine learning model that could both detect and depend on many signals from social media behavior to predict depression early. They developed a crowdsourcing approach to solve the challenge of creating a ground-truth dataset. Commenters were recruited through Amazon Mechanical Turk and were required to complete the Center for Epidemiologic Studies Depression scale (CES-D) test. Individuals were presented with a questionnaire about their depressive symptoms, past history, and current state. The Mechanical Turkers who answered the questionnaire were asked for their Twitter username, which could then be used to extract their Twitter feed, resulting in a depressed/non-depressed baseline dataset. Using features obtained from both the content of the tweets and network features such as the number of followers, a machine learning classifier was then created on the depressed/nondepressed data. This classifier was applied to a large dataset of geolocated Twitter data from the United States and found to have a high positive association with depression statistics from the Centers for Disease Control and Prevention (CDC). De Choudhury et al. presented research that examined more than 2 million tweets from 476 users to see if they could predict depression from them. SVM classifiers using a collection of behavioral features, such as the use of pronouns, curses, and depressive words; tweet responses; and posting time and frequency, produced the best results.

Chen et al. [4] addressed mental health issues in publicly available Twitter data. They quickly and inexpensively obtained data from a wide range of mental health disorders to identify various symptoms of mental illness such as depression, bipolar disorder, and seasonal affective disorder. A Linguistic Inquiry Word Count (LIWC) analysis of each disorder was used to determine the extent to which each disorder group differed from a control group. This sought to replicate previous findings for depression and provide new insights for bipolar disorder, PTSD, and SAD. There are two linguistic models: (1) a standard unigram LM to examine the likelihood of each word and (2) a character 5-gram LM to examine sequences of up to five characters. McDonnell et al. [21] developed several classifiers that can be used to distinguish each group from the control group. Correlations between the analyses and the classifiers were then examined to identify relationships between them. This provided insight into measurable and important signals of mental health on Twitter.

Lin et al. [16] presented the study of data from four microblogs and compared the performance of their proposed four-layer DNN with traditional stress detection methods such as Random Forest, SVM, and Naive Bayes. They used three pooling approaches to evaluate performance for each model: max-pooling, mean-over-instance, and mean-over-time. Depending on the pooling approach, each model performed well or poorly. DNN, on the other hand, performed best when using mean-over-time pooling. 
Using the NLP method of dependency parsing, Neuman et al. [27] developed another approach called "Pedesis" that searches web pages for metaphorical relations in which depression is embedded and retrieves the corresponding conceptual domains. The domain is used to define phrases or words that have been used as metaphors for mental illness.

Hidden patterns and high-dimensional features are often used to help the neural network learn a unique representation of the feature space [29]. The trained network then applies the learned features to the conditional distribution of input vectors in the computer. Different neural network architectures are proposed for domain-specific applications. The weights are updated. The network is necessary to reduce the loss in supervised learning, which is a nonlinear optimization problem. To maximize the loss, the weight and bias values are used. The methods are mostly based on the gradient descent method. Gradient-based methods start with random points for each input vector. They then perform multiple iterations (epochs) for a collection of instances (batches). Loss was determined by computing the trainer's non-linear objective function for the loss values and gradient. The weights are then adjusted to reduce the loss function [29]. The loss is gradually reduced until it reaches the convergence point or the best local minimum. Hidden layers and the structure of the architecture give neural networks their predictive power. Proper selection of multiple layers, architecture structure, layers, and hyperparameters helps in solving complex problems. The network training [6] is used to generate a higher-order representation of the vector of input features. A higher feature representation is learned, which supports generalization and increases predictive power. In modern neural network research, the network with the lowest computational complexity and the highest predictive power is chosen. In the last two decades, a variety of architectural proposals have been made [6].

The hidden layers, layer types, shapes, and connections between layers are the main differences between the designs [34]. The method developed by Wainberg et al. learns higher-dimensional features from tabular data [36]. The Convolutional Neural Network (CNN) learns a feature embedding from the image pixels. The pixel data and the variance between them improve the learning and prediction capabilities of the network. The network [36] benefits from the translation-stable pixels. Much research has been done on learning and inference in the visual information processing system, including applications in wildlife [9], X-ray scans [32], and self-driving vehicles. The recurrent neural network (RNN) architecture processes the input features with different gates, which mainly depend on the lost feature [2]; the fixed-length vector occasionally loses necessary details. The alignment of input and output vectors is another difficulty in RNN encoder and decoder architecture. The values of adjacent features affect the sequence. Their proposed novel network called attention mechanism is another version of RNN. It uses the attention method of the input vector to assign specific weights to the selected inputs. The decoder uses the position with the context vector and associated weights for the higher feature representation, which depends on the prioritized importance and position of the relevant data. The weights for the RNN model for the predictions [1], attention weights, and context vector are then trained based on the architecture and feature representation. There are several network versions for the attention mechanism, including a soft, hard, and global design.

$\mathrm{Xu}$ et al. construct the context vector at hard attention by sampling the hidden states [37]. Hard attention lowers the computational cost; however, design adaptation is challenging because architectural convergence is difficult. Another version proposed by Luong et al. [19] is local and global attention. Global attention is a middle ground between soft and strong attention. The model selects an attention point for each input stack. This helps to achieve fast convergence. The prognostic function is used to learn the location of the interest vector in the local attention mechanism. Both local and global attention are computationally intensive and must be selected based on domain-specific statistical analysis. 
Table 1. Critical Analysis of the Methods

\begin{tabular}{|l|c|c|c|c|c|}
\hline Paper & Dataset & Method & Machine Learning & Unsupervised & Adaptive Learning \\
\hline$[38]$ & Online Forum & Feature based & Naive Bayes, Maximum Entropy, and Decision Tree & No & No \\
\hline$[8]$ & A Thin Line (ATL) 7,147 stories & Feature based & Stacking & No & No \\
\hline$[7]$ & Amazon Mechanical Turk 2 million & Text features & SVM & No & No \\
\hline$[16]$ & Twitter/Micro-blog & Location-based analysis & CNN & No & No \\
\hline$[2]$ & Amazon Mechanical Turk & Synonyms & Attention network & 84.3 \\
\hline
\end{tabular}

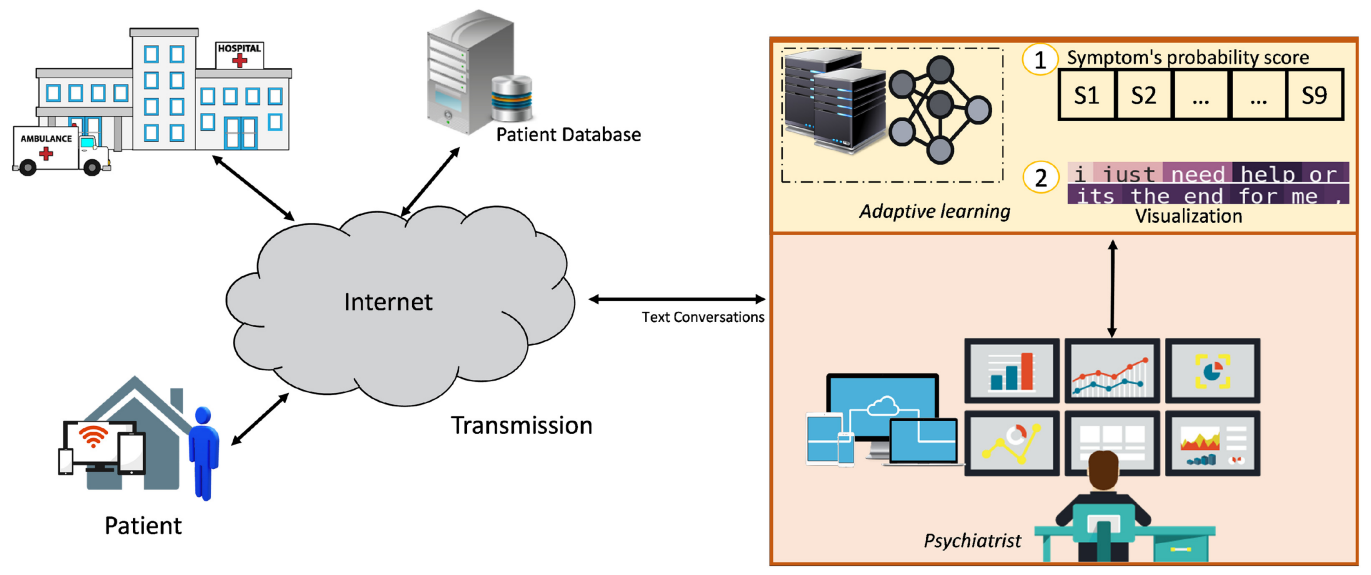

Fig. 1. Adaptive online intervention based on patient-authorized text.

As mentioned in Table 1, most of the works used the text-based feature extraction method. However, unsupervised learning methods with adaptivity remained an open research area; most of them used the text features and then applied the statistically based machine learning method. However, given the data-driven nature of the modern application, modern tools need an adaptive method that can account for the changes in data points as they interact with the environment.

\section{HIERARCHICAL ATTENTION-BASED ACTIVE LEARNING}

This work uses natural language processing (NLP) and attention-based in-depth entropy active learning to extract depression symptoms in mental health interventions. The flow of the online adaptive intervention is shown in Figures 1 and 2. For this purpose, this study proposes a technique based on synonym expansion using semantic vectors. Many researchers have used the unique vector space to compare two similar embeddings. However, we used the custom dataset to train the attention network. We also used transfer learning to use the glove embedding as the backbone or feature extractor for the network before training. The physiatrist questionnaire and the text written by the patient are used to extract embedding vectors. The questionnaire is then used by the proposed seed term generation method and passed to the embedding. The text written by the patient, on the other hand, is subjected to the standard preprocessing step and then passed to the embedding. The two resulting embeddings are then used to label the nine different labels. For this purpose, we used cosine similarity to the PHQ-9 symptom score. The trained attention network improved performance by knowledge expansion.

\subsection{Psychometric Questionnaires (PQ)}

The standard approach to measuring depression is the PHQ-9 questionnaire [13]. Many researchers have used this approach to assign depression scores. The solution was then tested on PHQ-9 to measure the depression score and the authorized text of the patients [2]. The other method includes PHQ-2, which contains 2 items, and PHQ-15, which contains 15 somatic symptoms [14]. It 


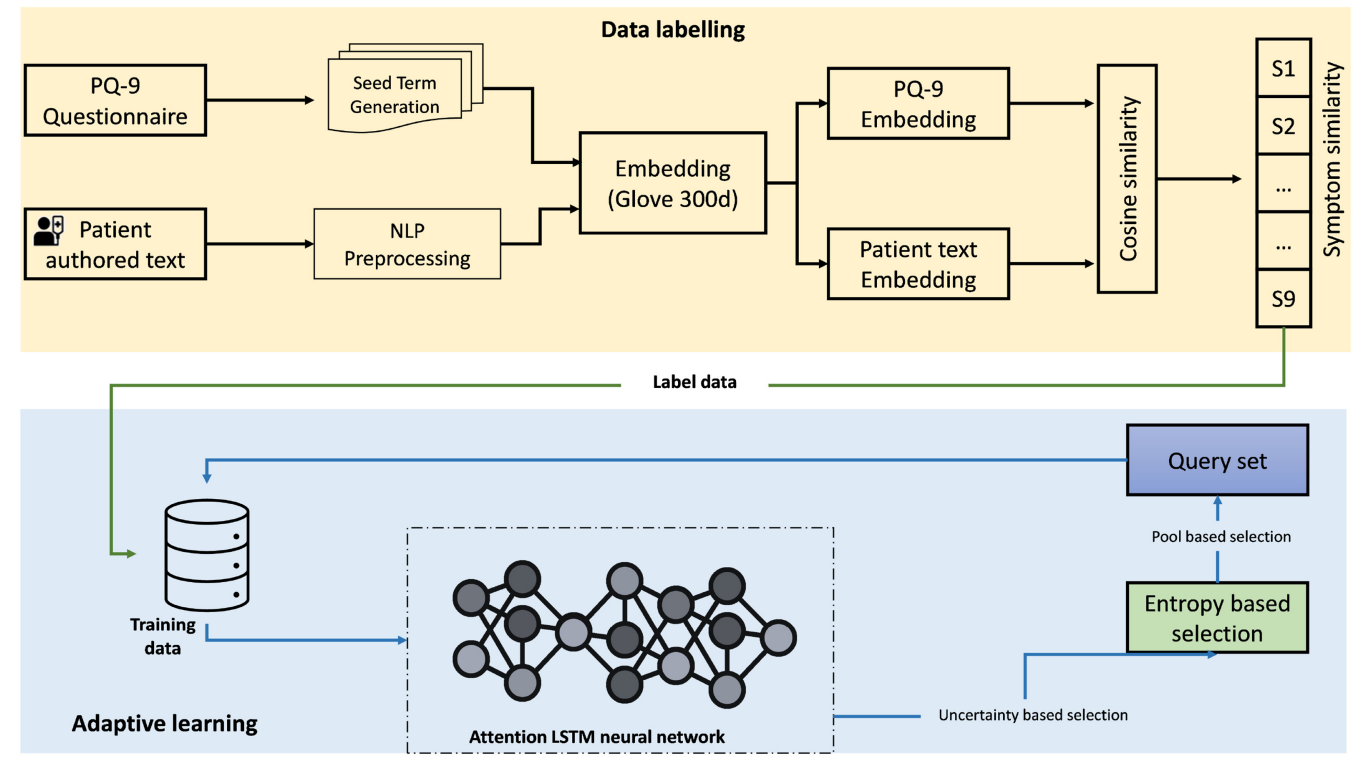

Fig. 2. Workflow for the proposed model.

is a complicated task to assess and diagnose mental health according to ICD10 [30] guidelines. The patient's mental health depends on many factors, such as family culture, previous treatments, society, childhood memories, work life, and daily routines. Therefore, during screening and history taking, psychiatrists closely examine the factors that trigger the mental condition. Generally, psychiatrists make a list of the patient's triggering events and mark the items. Based on the recorded points, the psychiatrists recommend some exercises. At the second visit, the psychiatrists use the standard questionnaire procedure based on the PHQ-9. The test is used to assess the clinical state of the patient's mental health. The test consists of different questionnaire schemes that include the type of symptoms, their cause, and treatment. A score is assigned by summing the frequency of symptoms. The resulting score describes the intensity of the psychological problems. For example, there are nine different symptoms; each symptom is further divided into mild, moderate, or severe conditions. The approach is called the Clinical Symptom Elicitation Process (CSEP) [30]. This research automates the process by using active learning and frequency response within the patient's textual conversation. Each symptom category is labeled using the patient's text and their clinical depression score. The PHQ-9 is a standard procedure in which the psychiatrist asks questions about triggering symptoms and observes the patient's responses to classify common symptoms into the classes listed below:

(a) Score 0: not at all

(b) Score 1: several days,

(c) Score 2: more than half the days

(d) Score 3: nearly every day

As a result, the DSM-V extracts nine different behavioral types. ${ }^{1}$ The behaviors describe the different mental disorders, which include sleeping, interest, concentration, and food disorder, as

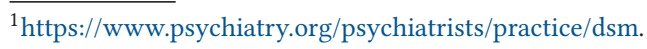


Table 2. PHQ-9 Questionnaire and Seed Terms of Each Symptom

\begin{tabular}{|c|c|c|}
\hline Symptons & PHQ-9 & Seed Terms \\
\hline S1 & little interest or pleasure in doing things & interest \\
\hline S2 & feeling down, depressed, or hopeless & feeling, depressed, hopeless \\
\hline S3 & trouble falling or staying asleep or sleeping too much & sleep, asleep \\
\hline S4 & feeling tired or having little energy & tired, energy \\
\hline S5 & poor appetite or overeating & appetite, overeating \\
\hline S6 & feeling bad about yourself or that you are a failure or have let yourself or your family down & failure, family \\
\hline S7 & trouble concentrating on things such as reading the newspaper or watching television & concentration, reading, watching \\
\hline S8 & moving or speaking so slowly that other people could have noticed or the opposite being so restless that you have been moving around a lot more than usual & moving, speaking, restless \\
\hline S9 & thoughts that you would be better off dead or of hurting yourself & dead, hurt, suicide \\
\hline
\end{tabular}

mentioned in Table 2 and the example document. ${ }^{2}$ The sum of the individual categories gives the total score, which describes the mental state of the patients.

\subsection{Word Embedding Using Emotional Lexicon}

Many researchers have exploited the sentiment extraction and detection mechanism. However, emotional knowledge-based (EKB) systems have not been studied. EKB systems use contextual embedding and emotional lexicons to build knowledge. The knowledge represents different sentiments and mental states. We used the Glove embedding for each word in the patient text [31]. The text is expanded using the seed terms for each questionnaire, which were handpicked based on the sentiments the questionnaire carries, as mentioned in Table 2. Then, we applied WordNet to the extracted words to find the synonyms $[23,25]$. Then, for each synonym, we found the corresponding hypernyms, hyponyms, and antonyms. We also expanded the words in the patient text by using the five most similar words based on the Glove embedding. Our empirical results show that the top five words contain most of the sentiment and context of the questionnaire. In this way, we extend the authorized text and the questionnaire to build the context for the behavior type. After that, we trained the Glove model to enhance the knowledge of the embedding before training. The custom embedding method is used to take advantage of transfer learning and expand the corpus by using word sense. The reason for this step is that most embeddings are based on the open source data; i.e., (Wikipedia texts) and sentiment knowledge (Twitter data) are trained. For instance, in the context of sentiment, the words happy and sad convey the sentiment of emotions. However, both words convey different meanings in relation to the PHQ-9 questionnaire. Therefore, word sense expansion is required for the data labeling.

\subsection{Preprocessing}

We compare the sentence (text written by the patient) with the PHQ-9 symptoms (S1-S9) and find the most similar symptoms. We preprocess the sentence to remove the inconsistent words. We first convert the text to UTF-8 format for consistency and then convert all words to lowercase. After that, we remove all extra spaces between sentences. Then we remove the irregular symbols $\left(\#,+,-,{ }^{*},=\right.$, HTTP, HTTPS) that have no meaning. Then we remove the slang and short forms of words; e.g., won't is replaced by will not, can't is replaced by cannot, and so on.

\subsection{Depression Embedding}

We extract the partial speech (noun, verb, adverb, and adjective) from the texts. The reason is that the mentioned parts of speech have sentimental meanings. The corpus $T$ consists of the set of documents $T=\left\{t_{1}, t_{2}, \ldots, t_{n}\right\}$ (Algorithm 1, line 2). As mentioned in Algorithm 1, we performed text processing (as mentioned in Section 3.3) and extracted part of speech. We used the WordNet to extract the synonyms, antonyms, hypernyms, and physical meaning for each extracted part-of-speech word (Algorithm 1, lines 3 to 8 ). The extended words consist of the sentiment set $S=\left\{s_{1}, s_{2}, \ldots, s_{K}\right\}$, which is combined into the set $T$ as mentioned in (Algorithm 1, line 9). The extended $T$ consists

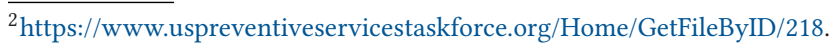




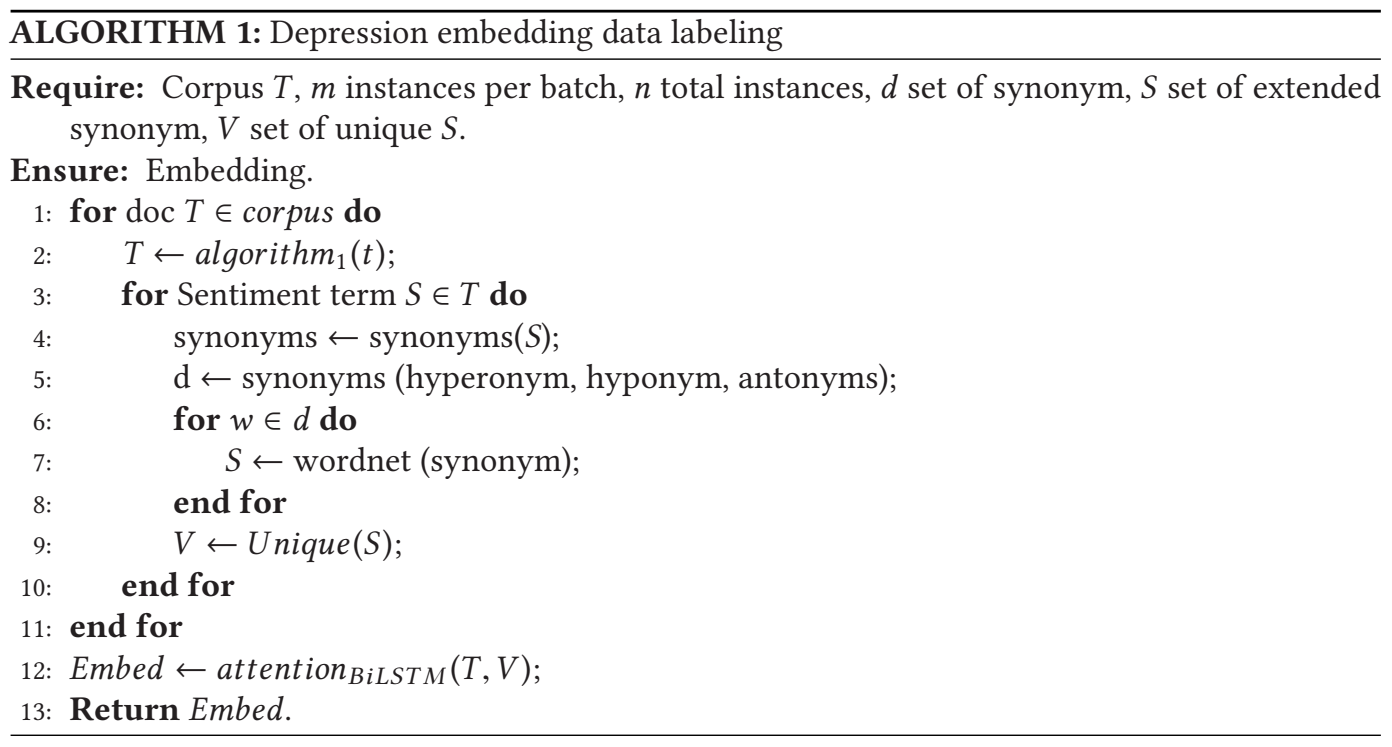

of the sentiment domain-specific corpus, where $s_{k}$ represents the sentiment words for each word in the text document (Algorithm 1, lines 3 to 5). The unique term of $T$ is stored in the vocabulary set $V_{S}=\left\{v_{1}, v_{2}, \ldots, v_{m}\right\}$ collected. For each $V_{m}$, the proposed embedding mapped the word representation in the latent space such that emotion-related words are closer to each other and non-related words are far from them; i.e., $T=\left\{t_{1}, t_{2}, \ldots, t_{n}\right\} \in \mathbb{R}^{n \times \delta}$, where $\delta$ is the dimension of the word vector and $n$ is the size of the vocabulary (Algorithm 1, line 12). We used the global attention model, which uses the intermediate representation of hard and soft attention [19]. For each batch of training patterns, the attention points are selected individually. Then, local attention learns the attention vector from the predictive function. Since the neighbor words affect the sequence of the texts, we used the predictive function to select the attention point. This method helps to converge quickly. The learned word vector representation yields the vector representations, $T_{v}=\left\{T_{v_{1}}, T_{v_{2}}, \ldots, T_{v_{n}}\right\}$.

\subsection{Data Labeling}

As shown in Figure 2, the embedding discussed in Section 3.4 is used for the data labeling task. Given two texts $P Q-9$ questionnaire and patient authorized text, we convert them into two vectors $\mathbf{t}$, e, where both vectors represent the sentiment latent representation. Then, we measure the similarity of the patient-authorized vector $\mathbf{e}$ with all symptoms of the extended latent representation (S1-S9). The output of the model is the multiclass prediction into nine different symptoms. In this way, we have nine different similarity values for each text. Then we label the data with the highest similarity. In this work, we used cosine similarity to compare these two vectors as defined below:

$$
\cos (\mathbf{t}, \mathbf{e})=\frac{\mathbf{t e}}{\|\mathbf{t}\|\|\mathbf{e}\|}=\frac{\sum_{i=1}^{n} \mathbf{t}_{i} \mathbf{e}_{i}}{\sqrt{\sum_{i=1}^{n}\left(\mathbf{t}_{i}\right)^{2}} \sqrt{\sum_{i=1}^{n}\left(\mathbf{e}_{i}\right)^{2}}} .
$$

\subsection{Adaptive Learning}

In this work, we extended learning to include the adaptive method. We have the labeled dataset from the Section 3.5 approach and shown in Figure 2. The entropy-based method is used to select the data distribution from the unlabeled dataset as shown in Figure 2. The model uses the attention 
mechanism to learn pattern recognition from a small collection of examples. The initial training set consists of a modest amount of data and is selected for inclusion in the training set using an entropy-based algorithm. The entropy-based methodology presented in this chapter determines the number of points in a pool based on its size. The pool point is a unique set that is updated on a cycle-by-cycle basis. The selected point is added to the training set and an alternative model is trained based on the new points. The repetition of the phases allows a gradual progress with the training set and the meaningful points. The proposed technique can reduce the data annotation effort and generalize the machine learning system [33].

\subsection{Deep Learning Model}

fasttext: We used the baseline model fasttext. This model uses embedding tokens in the distributed representation space [22]. Each word representation is then averaged to form a text representation. Then, the softmax function is used to calculate the probability distribution of the nine symptoms. After that, we used the cross-entropy loss function to calculate the loss for each batch. The model does not consider the word order in the word representation. For this reason, we used the n-gram features to calculate the partial information about the sentimental words. When the number of classes is very large, the computational cost of the softmax increases exponentially, while the hierarchical softmax is used to speed up the training process.

Bidirectional LSTM: For the sequential tasks, we used the gated RNN architecture. The RNN architecture is based on the memory method that helps in classifying the symptoms representing individual sentiments [1]. We used the element-wise averaging method in the hidden layer. The model used the two inputs, i.e., the original input vector and the second, inverted copy of the input vector. This gives additional context to the network.

Bidirectional LSTM with Global Attention: In bidirectional LSTM, the attention layer was used to increase the importance of the sentimental words. A more extensive network can help to achieve generalization. For each batch, the attention layer selects the points. Then, local attention learns the attention vector from the predictive function. This helps the model to converge quickly as weighted words help to classify the text more correctly. In entropy learning, the model adapts and improves its performance by increasing the number of labeled data points.

Hierarchical Attention Network (HAN): In this architecture, two levels of attention were applied, namely the word level and the sentence level [10]. Each word in the sentence is encoded into a word vector (Glove embedding) in the distributed vector space. The bidirectional gated recurrent neural network is used. By concatenating the vectors from two directions, it forms the vector representation of the sentence as mentioned in Equation (2):

$$
\begin{aligned}
x_{i t} & =W_{e} w_{i t}, t \in[1, T], \\
\vec{h}_{i t} & =\overrightarrow{\operatorname{GRU}}\left(x_{i t}\right), t \in[1, T], \\
\overleftarrow{h}_{i t} & =\overleftarrow{\operatorname{GRU}}\left(x_{i t}\right), t \in[T, 1]
\end{aligned}
$$

At the sentence level, sentence attention is used, which is similar to word attention, as mentioned in the Equation (3). It measures the importance of the sentence-level vector among sentences. The contextual information of the sentence is maintained between sentences:

$$
\begin{aligned}
\vec{h}_{i} & =\overrightarrow{\operatorname{GRU}}\left(s_{i}\right), i \in[1, L] \\
\overleftarrow{h}_{i} & =\overleftrightarrow{\operatorname{GRU}}\left(s_{i}\right), t \in[L, 1]
\end{aligned}
$$


Table 3. Experimental Setup

\begin{tabular}{c|cc}
\hline Device & Intel Core i7-9700K & RTX 2070 \\
Coffee lake & Turing (TU106) \\
Base clock & $3.6 \mathrm{Ghz}$ & $1410 \mathrm{Mhz}$ \\
Boost clock & 4.6 & $1710 \mathrm{Mhz}$ \\
Total cores & 6 & 2304 \\
Memory & $16 \mathrm{~GB}$ & $8 \mathrm{~GB}$ \\
Memory bandwidth & $32 \mathrm{~GB} / \mathrm{s}$ & $448 \mathrm{~GB} / \mathrm{s}$ \\
Deep learning library & Sklearn & Tensorflow \\
Language | library & Python 3.8 & CUDA $8.0 \mid \mathrm{CUDNN}$ \\
\hline
\end{tabular}

Table 4. Statistical Summary of the Training and Testing Sets

\begin{tabular}{lc}
\hline \multicolumn{1}{c}{ Type } & Statistics \\
\hline Corpus size (number of posts collected) & 15,044 \\
Number of sentences & 133,524 \\
Average sentences per post & 8.87 \\
Average words per post & 232 \\
Training set size (number of posts) & 14,944 \\
Testing set size (number of posts) & 100 \\
\hline
\end{tabular}

\section{EXPERIMENTAL RESULTS}

In this study, we used the Table 3 processing configurations. As described in the previous section, we first performed the preprocessing described in Section 3.3, then created the custom depression embedding (Section 3.4), and then performed the data labeling (Section 3.5). The labeled data is then used by the adaptive learning method (Section 3.6). We used the cross-entropy-based loss function. For evaluation, we used the true-positive rate $(T P R=T P /(T P+F N))$ and the falsepositive rate $(F P R=F P /(F P+T N)$ as performance measures. We also used precision, recall, and $\mathrm{f}$-measure to evaluate classification performance. For each deep learning architecture, we used the optimizer Adam [12], and the tuning of the hyperparameters is done using the adaptive learning rate starting from 0.0005 .

At the same time, other models tend to overfit because they perform well in the training set but not in the development and test sets. To avoid overfitting, three precautions were taken. First, the model was run over a longer period of time (1,000 epochs). Second, the concept of early stopping procedures was used to store the model incrementally. Third, a gradient pruning approach was used to ensure and remove gradient difficulties [5].

\subsection{Dataset}

We used the dataset that can be accessed through the online forum, website, and social media site [25]. The Amazon Mechanical Turk ${ }^{3}$ method is used to label the text [25]. The other labeling is done using the proposed method. In the labeling, the degree of depression was given in nine symptoms; i.e., 0 means not depressed, 1 means mildly depressed, 2 means moderately depressed, and 3 means severely depressed [25]. However, we convert to multi-label assignment, where the presence of each symptom means 1 and the absence means 0 . The collected data are listed in Table 4.

$\overline{{ }^{3} \text { https://www.mturk.com/. }}$ 


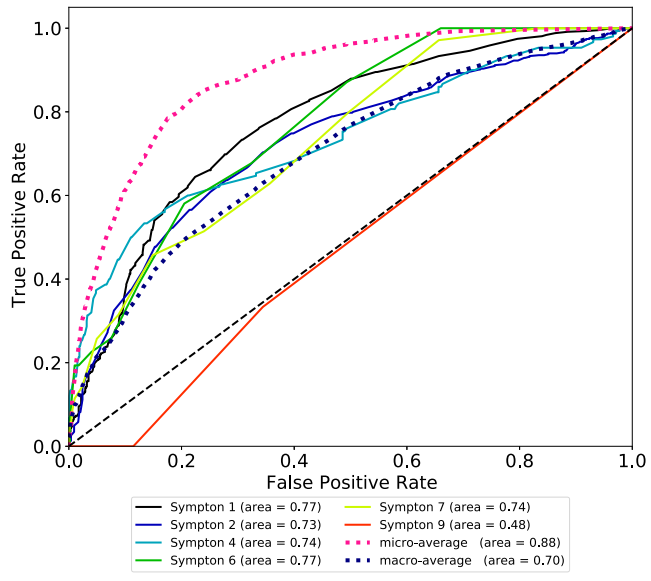

(a) Receiver operating characteristic

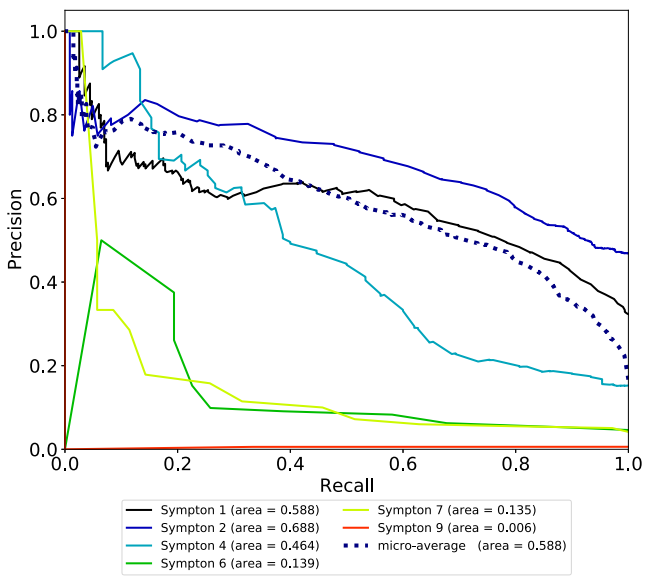

(b) Precision-recall curve

Fig. 3. Performance of fasttext model.

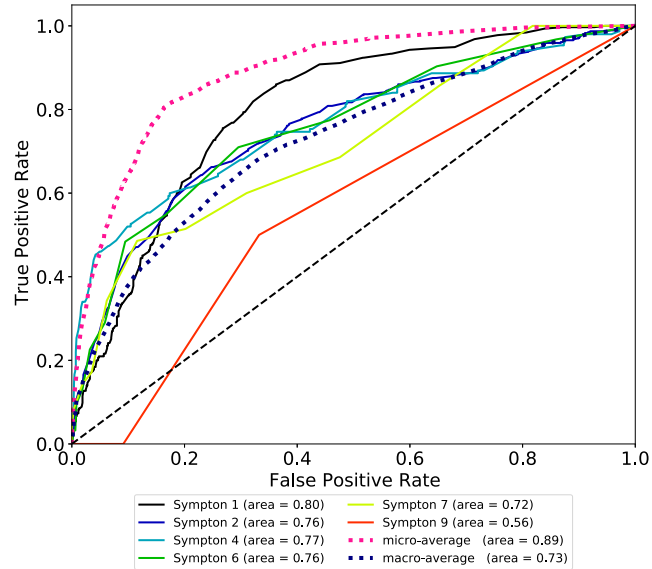

(a) Receiver operating characteristic

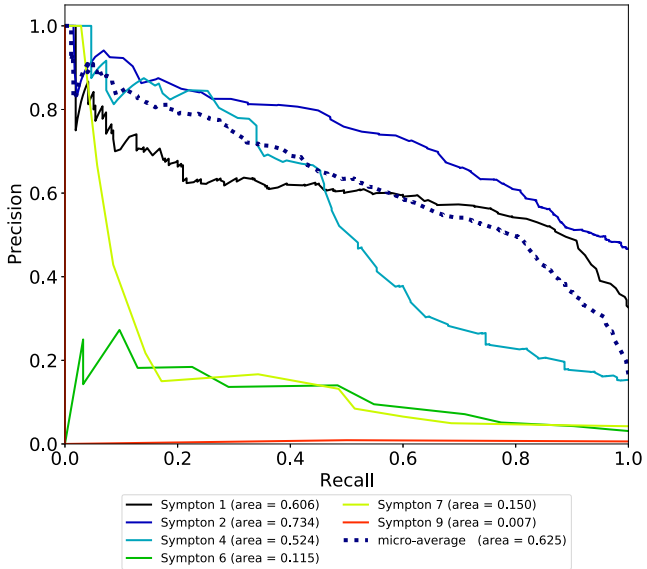

(b) Precision-recall curve

Fig. 4. Performance of Bidirectional LSTM model.

Figure 3 shows the results of the fastest base model. The trend graph shows that the model overfits as the upper left corner of the training, development, and test set converges on the precisionrecall curve. This is because the model uses the representation of a word regardless of its context. Words contain different meanings when combined with neighboring words. Since the word order and contexts are ignored, embedding them in a single representation can affect the meaning of the word. For effective embedding, the method should accept different word meanings and capture them effectively even if the means are present in the unbalanced training corpus.

The bidirectional LSTM model achieved a high precision-recall curve value of 0.88 , as mentioned in Figure 4. The bidirectional approach runs forward and backward, resulting in capturing the context. The hidden states preserve and store the relational meaning of the word. This leads to a low number of false positives. Embedding performed better due to the capture of word meaning. However, as the length of the text increases, long-range dependencies affect mode regularization. 


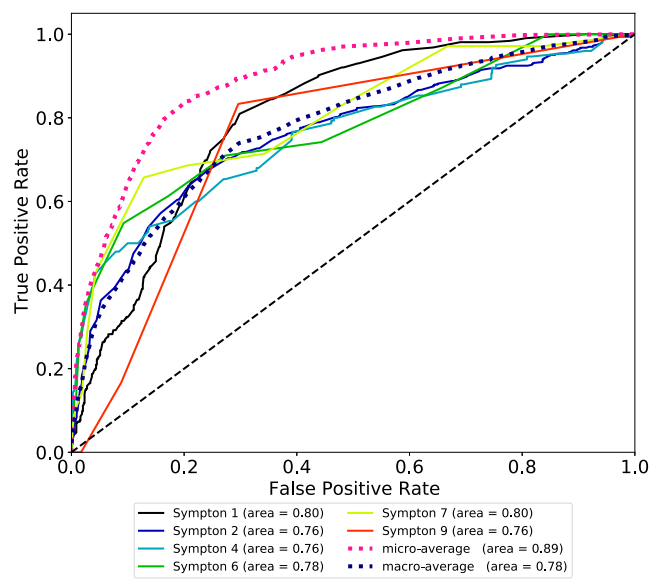

(a) Receiver operating characteristic

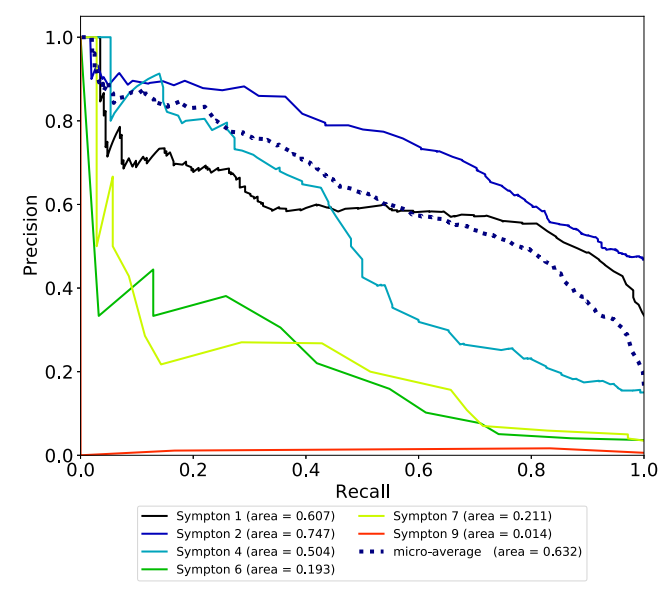

(b) Precision-recall curve

Fig. 5. Performance of Bidirectional LSTM with attention model.

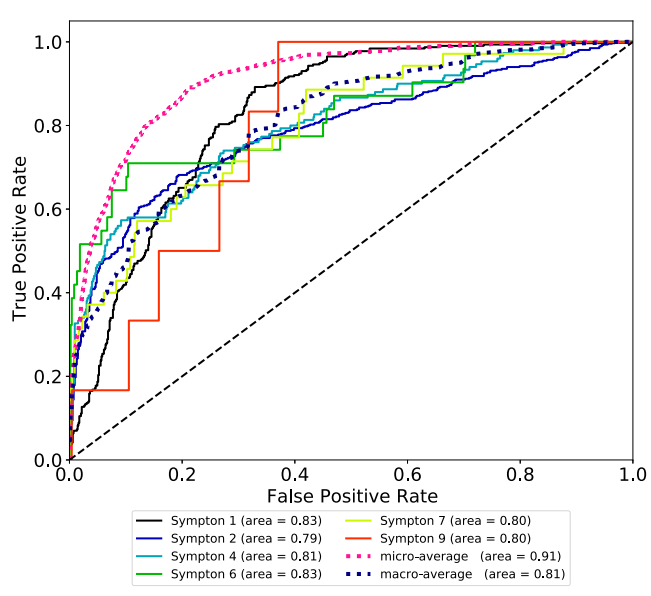

(a) Receiver operating characteristic

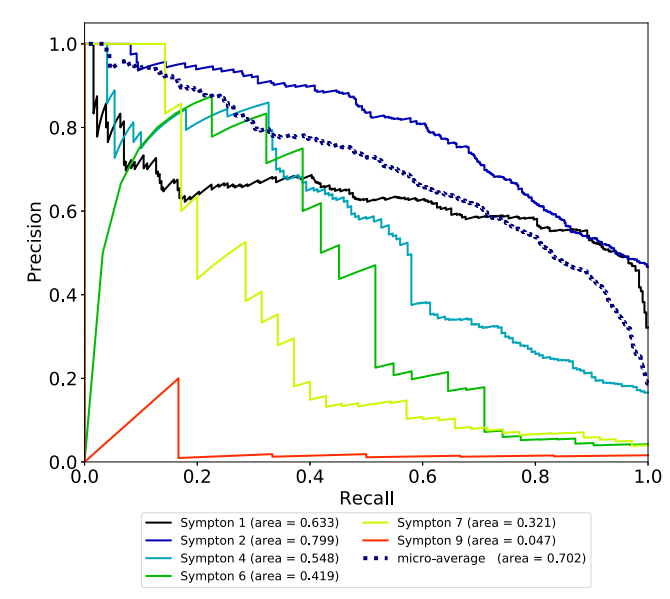

(b) Precision-recall curve

Fig. 6. Performance of hierarchical attention model.

In some batches, the models are unable to classify symptoms in lower classes. Due to the increasing length of the text and the overlap of sentiments, the model does not perform optimally.

In Figure 5, bidirectional LSTM with attention performed better than fasttext and bidirectional without attention. The weights chosen per batch and the position of the attention mechanism help to learn the word segmentation better. Sentence segmentation with word meaning helps to improve the results. This indicates that the attentions with position prediction function capture the temporal variations of the sentiment words. The reduced error in the training and development set with respect to the test set indicates that the model generalizes better. The high positive rate and reduced false-negative rate indicate that global attention helps to increase the training data. In Figure 6, the hierarchical attention structure performed better than bidirectional LSTM with attention. This indicates that local word position helps to learn emotional segments, and sentence attention indicates that the model learns context better. 


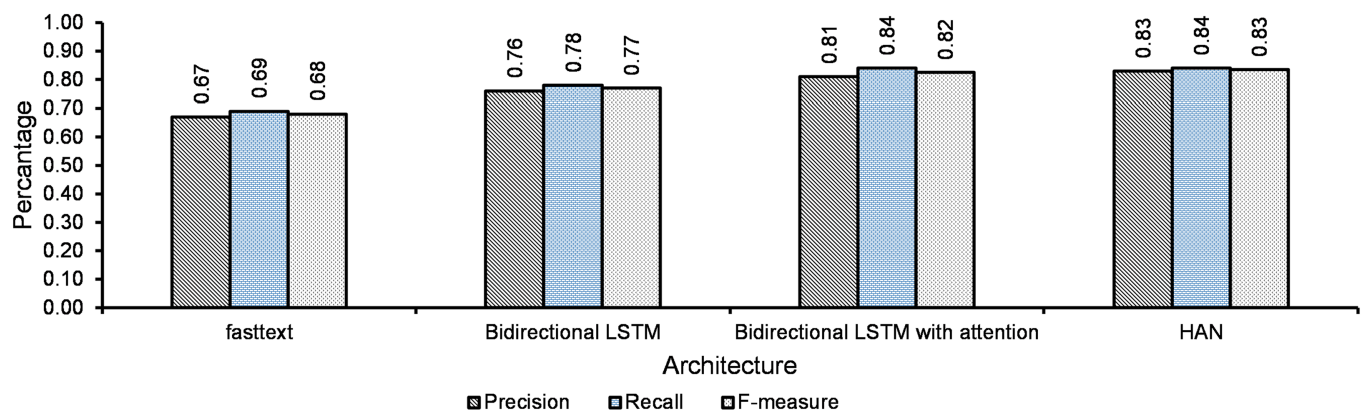

Fig. 7. Models' comparison by using the Glove embedding without word sense extension using cross-validation.

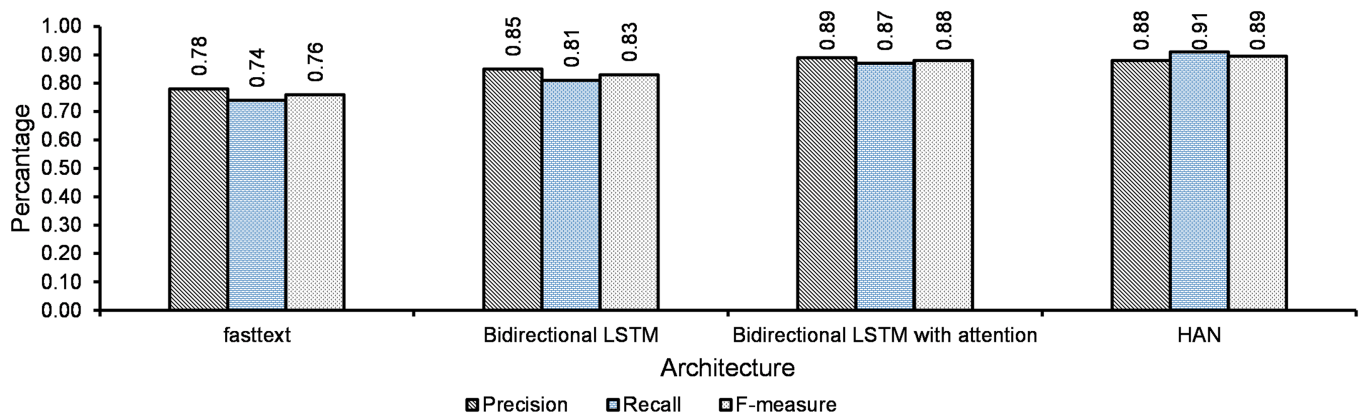

Fig. 8. Models' comparison by using the proposed words sentiment extension technique using cross-validation.

As mentioned in Figures 7 and 8, the attention method improves the results by the positional prediction function of the words. At the same time, the word sentiment knowledge expansion increases the overall performance of the model. In adapted learning, uncertainty sampling helps to identify the unlabeled instances near the decision boundary. The identification is based on the confidence of the model. The higher the confidence, the higher the chance of being included in the training cycle. As the results show, we used the entropy-based method to capture instances with a knowledge expansion of word sense by calculating how much the confidence differs from the predicted instances. This helps the psychiatrist to identify the trigger for the emotional feeling and its relation to the symptoms. The word and sentence attention of the HAN model helps indicate the words that help classify the symptoms. The model can be used as a computer-aided method for intervention via the Internet and helps the psychiatrist evaluate the notes.

The goal of this study is to use a semantic clustering technique to increase the number of instances that can be trained. The technique helps in generalizing the learning system by reducing the data annotation duties. The semantic vectors are clustered based on the semantic information extracted from the context in which they appear. Based on the semantic information, the result similarity metrics help to select a subset of unlabeled text. This technique selects the unlabeled text from the active learning mechanism cycle and includes it in the following cycle. The technique uses the new training points to update the model training. The cycle is repeated until the best solution is found. At this point, all unlabeled text is converted into the training set. The batchbased model, together with the attention network, can extract the important works that contain emotional meaning and association with the output class. The aggregation layers help to obtain the 
Table 5. Proposed Model Compared with and without Data Extension with Cross-validation Score

\begin{tabular}{|l|c|c|}
\hline \multicolumn{1}{|c|}{ Evaluation } & \multicolumn{2}{c|}{ F1 Score } \\
\hline \multicolumn{1}{|c|}{ Method } & Without Word Sense Extension & With Word Sense Extension \\
\hline fasttext & 0.68 & 0.76 \\
\hline Bidirectional LSTM & 0.77 & 0.83 \\
\hline Bidirectional LSTM with attention & 0.82 & 0.88 \\
\hline HAN & 0.83 & $\mathbf{0 . 8 9}$ \\
\hline
\end{tabular}

Table 6. Entropy-based Selection

of the HAN Model with Percentage

of Data Used

\begin{tabular}{cc}
\hline Percentage of Data & F1 Score \\
\hline 20 & 0.81 \\
40 & 0.85 \\
60 & 0.88 \\
80 & 0.89 \\
100 & 0.91 \\
\hline
\end{tabular}

Table 7. Cross-validation of the HAN Model

\begin{tabular}{lccc}
\hline Folds & Precision & Recall & F1-measure \\
\hline 2-Fold & 0.71 & 0.74 & 0.72 \\
3-Fold & 0.77 & 0.78 & 0.77 \\
5-Fold & 0.75 & 0.76 & 0.75 \\
10-Fold & 0.88 & 0.91 & 0.89 \\
\hline
\end{tabular}

representation of these informative words in the vectors. The process is used to detect the emotional words to form the sentences and then combine the sentences to make the document or conversation detectable for the symptoms. Since some words are misleading and some are very important, the important words are weighted for their neighbors. The results show that the performance of the model can be further improved even with more vocabulary and grammatical permutations. The model performance can be further increased with entropy-based adaptive learning as indicated in Tables 5 and 6. The cross-validation results also show that the increase of data can further improve the HAN model, as shown in Tables 5 and 7. The reason is that compared to the previous method, the proposed model is able to learn the meaning behind the sequences and construct attention-based weights for a word vector. Then, representation is aggregated with other words to form the sentence vectors. This aggregated vector contains all semantic meanings and patterns to classify the model. The proposed system achieved 0.85 ROC, which shows that the semantic vectors for synonym expansion improve the training accuracy without affecting the results.

\section{CONCLUSION}

In this article, we used a method of labeling unstructured data to highlight psychiatrists' notes. Advanced deep neural architecture with active learning was used to enhance the training data by automatically discriminating the classes. The entropy-based advanced learning method helps in increasing the training data and thus scaling up data labeling tasks. The model extends word sense by using word-sentiment- and context-based knowledge. The sentiment-based vocabulary created then helps reduce the over-fitting and generalization problem. The hierarchical attention-based model with the active learning method helps to improve the embedding learning. Word attention 
helps to emphasize the emotionally triggering event and sentence attention helps to expand the context of the text. This tool can be used as an online adaptive intervention, and a psychiatrist is used as a tool for making recommendation notes during online sessions with the mentally ill patient.

\section{FUTURE WORK}

In the future, we will use the automatic selection of the pre-training embedding to improve the selection technique for the linguistic lexicon. The textual information from the patient-authorized text has a limited scope. This study will be extended to use fuzzy set and contrast mining for the patient's geographical, cultural, religious, and social background to incorporate broader knowledge of the associated conditions. Also, a learning similarity network is analyzed for the scalability of the model. This helps in developing a more robust model in terms of accuracy, reliability, and integrity of the data. By using the transformer network and similarity network analysis, the physiological assistance method can help improve data assessment by better understanding the patient.

\section{REFERENCES}

[1] Usman Ahmed, Jerry Chun-Wei Lin, and Gautam Srivastava. 2021. Fuzzy explainable attention-based deep active learning on mental-health data. In IEEE International Conference on Fuzzy Systems. 1-6.

[2] Usman Ahmed, Suresh Kumar Mukhiya, Gautam Srivastava, Yngve Lamo, and Jerry Chun-Wei Lin. 2021. Attentionbased deep entropy active learning using lexical algorithm for mental health treatment. Frontiers in Psychology 12 (2021), 471.

[3] Xiangmao Chang, Gangkai Li, Guoliang Xing, Kun Zhu, and Linlin Tu. 2021. DeepHeart: A deep learning approach for accurate heart rate estimation from PPG signals. ACM Transactions on Sensor Networks 17, 2 (2021), 1-18.

[4] Emily Chen, Kristina Lerman, and Emilio Ferrara. 2020. Tracking social media discourse about the COVID-19 pandemic: Development of a public coronavirus Twitter data set. JMIR Public Health and Surveillance 6, 2 (2020), e19273.

[5] Xiangyi Chen, Zhiwei Steven Wu, and Mingyi Hong. 2020. Understanding gradient clipping in private SGD: A geometric perspective. In Advances in Neural Information Processing Systems 33: Annual Conference on Neural Information Processing Systems.

[6] Kyunghyun Cho, Bart van Merrienboer, Çaglar Gülçehre, Dzmitry Bahdanau, Fethi Bougares, Holger Schwenk, and Yoshua Bengio. 2014. Learning phrase representations using RNN encoder-decoder for statistical machine translation. In The Conference on Empirical Methods in Natural Language Processing, Alessandro Moschitti, Bo Pang, and Walter Daelemans (Eds.). 1724-1734.

[7] Munmun De Choudhury, Michael Gamon, Scott Counts, and Eric Horvitz. 2013. Predicting depression via social media. In The International Conference on Weblogs and Social Media.

[8] Karthik Dinakar, Emily Weinstein, Henry Lieberman, and Robert Louis Selman. 2014. Stacked generalization learning to analyze teenage distress. In Proceedings of the 8th International Conference on Weblogs and Social Media.

[9] Grant Van Horn, Oisin Mac Aodha, Yang Song, Yin Cui, Chen Sun, Alexander Shepard, Hartwig Adam, Pietro Perona, and Serge J. Belongie. 2018. The INaturalist species classification and detection dataset. In IEEE Conference on Computer Vision and Pattern Recognition. 8769-8778.

[10] Yingren Huang, Jiaojiao Chen, Shaomin Zheng, Yun Xue, and Xiaohui Hu. 2021. Hierarchical multi-attention networks for document classification. International fournal of Machine Learning and Cybernetics 12, 6 (2021), 1639-1647.

[11] Spencer L. James, Degu Abate, Kalkidan Hassen Abate, Solomon M. Abay, Cristiana Abbafati, Nooshin Abbasi, Hedayat Abbastabar, Foad Abd-Allah, Jemal Abdela, Ahmed Abdelalim, et al. 2018. Global, regional, and national incidence, prevalence, and years lived with disability for 354 diseases and injuries for 195 countries and territories, 1990-2017: A systematic analysis for the global burden of disease study 2017. The Lancet 392, 10159 (2018), 1789-1858

[12] Diederik P. Kingma and Jimmy Ba. 2014. Adam: A method for stochastic optimization. arXiv Preprint arXiv:1412.6980 (2014).

[13] Kurt Kroenke, Robert L. Spitzer, and Janet B. W. Williams. 2001. The PHQ-9: Validity of a brief depression severity measure. Journal of General Internal Medicine 16, 9 (2001), 606-613.

[14] Kurt Kroenke, Robert L. Spitzer, and Janet B. W. Williams. 2002. The PHQ-15: Validity of a new measure for evaluating the severity of somatic symptoms. Psychosomatic Medicine 64, 2 (2002), 258-266.

[15] Tim M. H. Li, Michael Chau, Paul W. C. Wong, and Paul S. F. Yip. 2012. A hybrid system for online detection of emotional distress. In Intelligence and Security Informatics. 73-80. 
[16] Huijie Lin, Jia Jia, Quan Guo, Yuanyuan Xue, Qi Li, Jie Huang, Lianhong Cai, and Ling Feng. 2014. User-level psychological stress detection from social media using deep neural network. In ACM International Conference on Multimedia. ACM Press.

[17] David E. Losada and Pablo Gamallo. 2018. Evaluating and improving lexical resources for detecting signs of depression in text. Language Resources and Evaluation 54, 1 (2018), 1-24.

[18] Daniel M. Low, Laurie Rumker, Tanya Talkar, John Torous, Guillermo Cecchi, and Satrajit S. Ghosh. 2020. Natural language processing reveals vulnerable mental health support groups and heightened health anxiety on Reddit during COVID-19: Observational study. Journal of Medical Internet Research 22, 10 (2020), e22635.

[19] Thang Luong, Hieu Pham, and Christopher D. Manning. 2015. Effective approaches to attention-based neural machine translation. In The Conference on Empirical Methods in Natural Language Processing, Lluís Màrquez, Chris CallisonBurch, Jian Su, Daniele Pighin, and Yuval Marton (Eds.). 1412-1421.

[20] Mario Gennaro Mazza, Rebecca De Lorenzo, Caterina Conte, Sara Poletti, Benedetta Vai, Irene Bollettini, Elisa Maria Teresa Melloni, Roberto Furlan, Fabio Ciceri, Patrizia Rovere-Querini, and Francesco Benedetti. 2020. Anxiety and depression in COVID-19 survivors: Role of inflammatory and clinical predictors. Brain, Behavior, and Immunity 89 (2020), 594-600.

[21] Michelle McDonnell, Jason Edward Owen, and Erin O'Carroll Bantum. 2020. Identification of emotional expression with cancer survivors: Validation of linguistic inquiry and word count. FMIR Formative Research 4, 10 (2020), e18246.

[22] Armand Joulin, Edouard Grave, Piotr Bojanowski, and Tomas Mikolov. 2017. Bag of Tricks for Efficient Text Classification. In Proceedings of the 15th Conference of the European Chapter of the Association for Computational Linguistics: Volume 2, Short Papers. 427-431.

[23] George Miller, Christiane Fellbaum, Judy Kegl, and Katherine Miller. 2009. WordNet: An electronic lexical reference system based on theories of lexical memory. Revue québécoise de linguistique 17, 2 (2009), 181-212.

[24] Julia Mühleck, Sigrid Borse, Eva Wunderer, Bernhard Strauß, and Uwe Berger. 2019. Online-Befragung zur Bekanntheit von Angeboten zur Aufklärung, Prävention, Beratung und Nachsorge bei Essstörungen. Prävention und Gesundheitsförderung 15, 1 (2019), 73-79.

[25] Suresh Kumar Mukhiya, Usman Ahmed, Fazle Rabbi, Ka I. Pun, and Yngve Lamo. 2020. Adaptation of IDPT system based on patient-authored text data using NLP. In IEEE International Symposium on Computer-based Medical Systems. 226-232.

[26] Suresh Kumar Mukhiya, Jo Dugstad Wake, Yavuz Inal, Ka I. Pun, and Yngve Lamo. 2020. Adaptive elements in internet-delivered psychological treatment systems: Systematic review. Journal of Medical Internet Research 22, 11 (2020), e21066.

[27] Yair Neuman, Yohai Cohen, Dan Assaf, and Gabbi Kedma. 2012. Proactive screening for depression through metaphorical and automatic text analysis. Artificial Intelligence in Medicine 56, 1 (2012), 19-25.

[28] Antoine Neuraz, Ivan Lerner, William Digan, Nicolas Paris, Rosy Tsopra, Alice Rogier, David Baudoin, Kevin Bretonnel Cohen, Anita Burgun, Nicolas Garcelon, et al. 2020. Natural language processing for rapid response to emergent diseases: Case study of calcium channel blockers and hypertension in the COVID-19 pandemic. Fournal of Medical Internet Research 22, 8 (2020), e20773.

[29] Giang Nguyen, Stefan Dlugolinsky, Martin Bobák, Viet D. Tran, Álvaro López García, Ignacio Heredia, Peter Malík, and Ladislav Hluchý. 2019. Machine learning and deep learning frameworks and libraries for large-scale data mining: A survey. Artificial Intelligence Review 52, 1 (2019), 77-124.

[30] World Health Organization et al. 1993. The ICD-10 Classification of Mental and Behavioural Disorders: Diagnostic Criteria for Research, Vol. 2.

[31] Jeffrey Pennington, Richard Socher, and Christopher D. Manning. 2014. Glove: Global vectors for word representation. In The Conference on Empirical Methods in Natural Language Processing. 1532-1543.

[32] Pranav Rajpurkar, Jeremy Irvin, Kaylie Zhu, Brandon Yang, Hershel Mehta, Tony Duan, Daisy Yi Ding, Aarti Bagul, Curtis Langlotz, Katie S. Shpanskaya, Matthew P. Lungren, and Andrew Y. Ng. 2017. CheXNet: Radiologist-level pneumonia detection on chest X-Rays with deep learning. CoRR abs/1711.05225 (2017).

[33] Christopher Schröder and Andreas Niekler. 2020. A survey of active learning for text classification using deep neural networks. CoRR abs/2008.07267 (2020). arXiv:2008.07267. https://arxiv.org/abs/2008.07267.

[34] Vivienne Sze, Yu Hsin Chen, Tien Ju Yang, and Joel S. Emer. 2017. Efficient processing of deep neural networks: A tutorial and survey. Proceedings of the IEEE 105, 12 (2017), 2295-2329.

[35] Emily A. Troyer, Jordan N. Kohn, and Suzi Hong. 2020. Are we facing a crashing wave of neuropsychiatric sequelae of COVID-19? Neuropsychiatric symptoms and potential immunologic mechanisms. Brain, Behavior, and Immunity 87 (2020), 34-39.

[36] Michael Wainberg, Daniele Merico, Andrew Delong, and Brendan J. Frey. 2018. Deep learning in biomedicine. Nature Biotechnology 36, 9 (2018), 829-838. 
[37] Kelvin Xu, Jimmy Ba, Ryan Kiros, Kyunghyun Cho, Aaron C. Courville, Ruslan Salakhutdinov, Richard S. Zemel, and Yoshua Bengio. 2015. Show, attend and tell: Neural image caption generation with visual attention. In Proceedings of the 32nd International Conference on Machine Learning (ICML'15) (FMLR Workshop and Conference Proceedings, Vol. 37). JMLR.org, 2048-2057.

[38] Lingling Xu, Ruyi Jin, Feifei Huang, Yanhui Zhou, Zonglong Li, and Minqiang Zhang. 2020. Development of computerized adaptive testing for emotion regulation. Frontiers in Psychology 11 (2020), 3340.

[39] Yifan Zhang and Xinglin Zhang. 2021. Price learning-based incentive mechanism for mobile crowd sensing. ACM Transactions on Sensor Networks 17, 2 (2021), 1-24.

Received 29 September 2021; revised 18 January 2022; accepted 15 February 2022 